\title{
Imprint Cytology for Intra-operative Assessment of Surgical Margins for Skeletal Tumours in COVID-19 Pandemic
}

\author{
Jagandeep Singh Virk ${ }^{1}$ (D) Poonam Bhaker ${ }^{2} \cdot$ Harsh Mohan ${ }^{3,2} \cdot$ Rajeshwar Singh $^{4}$
}

Accepted: 23 June 2020 / Published online: 29 June 2020

(C) Springer Nature Switzerland AG 2020

\begin{abstract}
In the current COVID-19 pandemic, tremendous pressure is been exerted on the existing health infrastructure of many developing nations. Limb salvage surgeries in bone and soft tissue sarcomas cannot be delayed beyond a certain time-period and constitute an orthopaedic emergency in certain situations. Evaluation of intra-operative surgical margins forms an important step especially in cases with planned close margins or intercalary resections. Techniques such as imprint cytology can be preferred over frozen sections for evaluation of the surgical margins especially in COVID-19 positive patients. The advantages it offers such as completion of the procedure within the operation room complex, no generation of aerosols, and almost equal sensitivity and specificity when compared with frozen section method do warrant a modification of current surgical practice in the current health crisis especially in resource-constrained nations. Awareness and better communication regarding the same from the pathologist to the surgical team will go a long way in conserving resources and avoid unnecessary exposure to potentially infected aerosols.
\end{abstract}

Keywords Sarcomas $\cdot$ Limb salvage $\cdot$ Margins $\cdot$ COVID-19

\section{Introduction}

Incidence of sarcomas of the bone is about 8 to 9 per million population per year and contribute to about $1 \%$ of all the cancers diagnosed worldwide $[1,2]$. Although a rare entity in

This article is part of the Topical Collection on Covid-19

Jagandeep Singh Virk

jaganvirk_09@yahoo.co.in

Poonam Bhaker

punam4_84@yahoo.co.in

Harsh Mohan

drharshmohan@gmail.com

Rajeshwar Singh

rsrajusingh62@gmail.com

1 Department of Orthopaedic Oncology, Paras Hospitals, Panchkula Haryana India

2 Oncquest Laboratories, Paras Hospitals, Panchkula Haryana India

3 Department of Pathology, GMCH, Chandigarh, Chandigarh India

4 Medical Oncology, Paras Hospitals, Panchkula Haryana India
Orthopaedic practice, appropriate management is paramount for these malignancies, considering the aggressive nature of few pathologies. Primary bone sarcomas such as Osteosarcoma, Ewing's sarcoma, and Chondrosarcoma can metastasize to lungs, bones, or bone marrow. A delay in the diagnosis and initiation of the appropriate treatment can be detrimental to the overall survival and prognosis of the patient.

In the current pandemic, COVID-19 cases are increasing at a tremendous rate world over and more than 2.3 lakhs persons have tested positive for COVID-19 in India itself, until the filing of this commentary [3]. The present health crisis is exerting tremendous pressure on the existing healthcare system of a developing nation like ours. Certain orthopaedic surgeries in situations like trauma, severe infections, and malignant tumours are certainly unavoidable which endanger the limb/life of the patients. In orthopaedic oncology, patients requiring surgeries for their sarcomas are also unavoidable beyond a certain period. Timing of the surgery and timely resumption of the chemotherapy post-surgery form an important part of sarcoma management and provide a good overall survival (OS) to such patients.

As part of the limb salvage surgeries - sometimes determining the status of the surgical margins - bony as well as 
soft tissue is quintessential for the operating surgeon intraoperatively. This involves transportation of fresh unfixed surgical specimens to the pathology labs where these are subjected to frozen section examinations. The process of frozen sections itself generates a lot of aerosols in the pathology lab and potentially creates another hazardous zone apart from the operation theatre (OT), especially when dealing with samples in a COVID-19-positive case.

A useful alternative for assessing the intra-operative surgical margins is imprint cytology (IC). The slides for cytological assessment can be prepared by the surgeon in the OT and examined by the pathologist in the OT complex itself. This circumvents the requirement for transportation of potentially hazardous tissue samples outside the OT complex and subjection to unnecessary aerosol-generating procedures like frozen sections. Many centres and hospitals still seem to be following the routine practice of obtaining frozen sections intra-operatively, despite these concerns due to lack of awareness regarding the same. Thus, better interaction between the orthopaedic surgeon and the pathologist and sensitization to such practices is the need of the hour in this COVID-19 crisis.

\section{Material and Methods}

\section{Imprint Cytology vs Frozen Section Examination for Gaining Intra-operative Margin Status in Sarcomas}

Intra-operative pathological consultation can be done by techniques such as frozen section and imprint cytology (touch imprint, crush, or scrape), each having its advantages and limitations. Both techniques are quick, provide reliable intraoperative provisional tissue diagnosis, and provide a reasonable assessment of the adequacy of surgical resection limits. This helps the surgeon to make an intra-operative decision regarding proceeding with further extension of surgical margins or not. The diagnostic yield, sensitivity, and specificity of both techniques for tumour detection in specimens or margins are almost comparable in several studies $[4,5]$.

\section{Technique for Imprint Cytology}

Direct imprints are prepared by pressing the microglass slide gently against the freshly cut surface of the specimen. Gliding movement is avoided. Smear is immediately fixed in alcoholbased fixatives and one may be left to air dry. These smears are stained by Toluidine blue, Diff quick stain, rapid haematoxylin and eosin (H\&E), and May Grunwald Giemsa (MGG) [6]. The technique of preparing the glass slides can be easily understood in detail by the Orthopaedic Oncosurgeon from the pathologist. The marrow margin status from the cut ends of the bone to confirm appropriate resection length, as well as soft tissue margin status, can be obtained.

\section{Disadvantages of Frozen Section Examination in the Current COVID-19 Crisis}

The technique of frozen section diagnosis has several disadvantages in the current COVID-19 crisis:

1. The main problem is the persistence of SARS-CoV-2 on inanimate surfaces such as cryostats; the virus can survive temperatures of $-20^{\circ} \mathrm{C}$, the temperature used for cutting of fresh-frozen sections [7].

2. The potential for extensive aerosol creation during the processing of the specimen. The cutting blade itself poses the highest risk of surface contamination and is required to be handled with extreme care.

3. Ideally, frozen sections have to be performed in a separate area away from the main laboratory workflow to minimise exposure risk to other staff.

4. Strict adherence to cryostat decontamination protocols has to be followed which takes a long-time and many laboratories have only one cryostat machine available for frozen sections.

Besides these disadvantages, the pathologist and their technicians have to work in proper PPE gear and wear protective eye gear and $\mathrm{N} 95$ face masks while preparing frozen sections. Many times, fogging over the protective face shield or the eye-

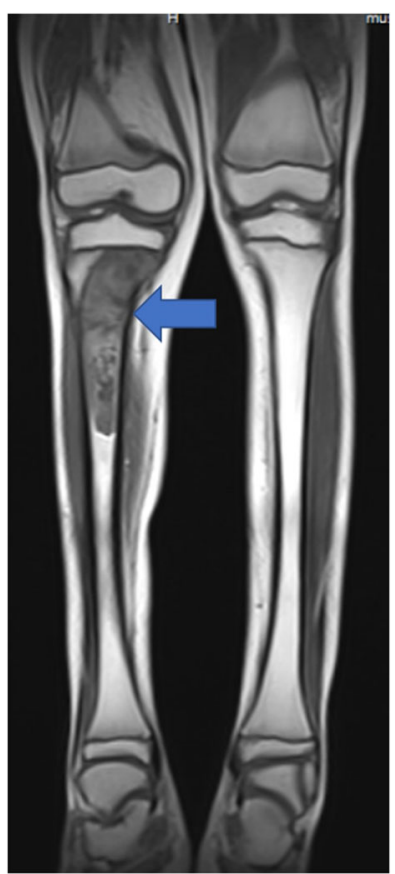

Fig. 1 T1 coronal MRI section showing a biopsy-proven Ewing's sarco$\mathrm{ma}$ in the proximal part of tibia in the left leg (Blue arrow). An intercalary joint-preserving surgical resection was planned for this patient. Intraoperative confirmation by frozen section/imprint cytology of negative resection margins is often required when the margins are planned close to the tumour 
gear can lead to difficulty in correct and timely interpretation of the frozen sections.

\section{Advantages of Imprint Cytology in the Current COVID- 19 Crisis for Gaining Intra-operative Resection Margin Status in Sarcoma Surgeries}

1. The sensitivity, specificity, negative predictive value (NPV), and diagnostic adequacy of imprint cytology are excellent and almost comparable with traditional methods like frozen sections [5].

2. No risk of aerosol generation or special requirement of a separate facility in the laboratory.

3. The average turnaround time is less than $20 \mathrm{~min}$ and comparable with frozen sections $[4,8]$.

4. The microscope can be placed in the OT complex and the pathologist be requested to examine the appropriately stained-glass slides in the OT complex itself thereby avoiding unnecessary transportation of specimens outside the OT complex.

5. Inexpensive.

\section{Discussion}

Limb salvage, wherever possible, has become the modality of choice for surgical treatment of primary malignant bone sarcomas. The success of a limb salvage surgery is heavily dependent on the quality of our surgical margins [9]. The resection of high-grade sarcomas is performed applying all the principles of sarcoma management and determining the quality of our surgical margins by the barrier concept as elucidated by Kawaguchi et al. [10]. Assessment of the extent of our surgical resection - bony/soft tissue - is usually planned preoperatively on a recent good-quality contrast-enhanced MRI with fine cuts and all sequences, CT, and radiographs. At times, use of intra-operative navigation system especially in resections around the pelvis is beneficial for confirming our bony resection margins. The availability of the same is a constraint at most centres.

In most situations, these tumours also have a soft tissue component, and confirming negative resection margins by the way of frozen sections is a standard protocol at most centres. This is usually imperative when our resection limits are very close to the tumour or the bony osteotomies are placed extremely close to the tumour as in intercalary resections while preserving the patient's joints (Fig. 1).

A method like imprint cytology which is accurate, quick, and safe in the current COVID-19 scenario for judging the resection margins status intra-operatively in oncosurgeries is warranted. The role of imprint cytology in other subspecialities of oncosurgery has also been widely reported. In a retrospective study of 100 cases of breast lumpectomy specimens, which underwent intra-operative imprint cytology for studying margin status, also concluded imprint cytology to be a reliable diagnostic tool for the evaluation of the status of lumpectomy margins in breast cancer patients. Studying the margins for any microscopic residual foci of tumour cells forms an important part of breast-conservation surgery. This study conducted at the Department of Pathology at University of Florida found intra-operative imprint cytology to have a sensitivity of $97 \%$, specificity of $99 \%$, with positive predictive value (PPV) of $84 \%$, and negative predictive value (NPV) of 99\% [11]. Another study on head and neck cancers, which constitutes one of the most commonly encountered cancers especially in South East Asia, concluded imprint cytology to be a reliable investigation for specific diagnoses and an adjunct to histopathology, particularly in developing countries. The overall diagnostic accuracy of imprint cytology in this study was $96.7 \%$ with a sensitivity and specificity of 96 and $100 \%$, respectively while PPV and NPV were found to be 100 and $84 \%$, respectively [12]. Inputs from other specialities have also highlighted the potential hazards associated with the use of frozen sections for intra-operative assessments in the present health crisis. The American Association of Ophthalmic Oncologists and Pathologists (AAOOP) in their Ocular Pathology recommendations during COVID-19 have also discouraged frozen section examinations and encouraged submission of fresh fixed specimens for imprint cytology [13]. Pathologists from some associations like the European Association of Urology have categorically asked the surgeons to refrain from the routine practice of asking for frozen sections, unless necessary. The guidelines from this association label the SARS-COV2 as extremely contagious and lethal and advice both pathologists and laboratory workers to be extremely cautious when handling fresh urological specimens [14]. Taking insights into other sub-specialities of oncosurgery, orthopaedic oncosurgery also needs to inculcate use of imprint cytology over frozen sections for studying surgical margins wherever feasible in the current health crisis.

\section{Conclusion}

Although COVID-19 testing is to be performed for suspected cases, the risk of cross-transmission in a hospital setting also catering to treatment of COVID-19-positive patients is also a potential problem. The hospitals, its OT's, and laboratories are potential hot zones for contracting COVID-19 infection. In such a scenario, our health system must act immediately, wisely, and support essential surgical care while protecting patients and staff and conserve valuable resources. Use of procedures like imprint cytology in the current scenario will not only conserve resources but also protect our pathology colleagues and labs from unnecessary aerosol-generating 
procedures like frozen sections for intra-operative assessment of surgical margins for sarcomas.

Authors Contribution JSV, PB, RS-To conception and design of commentary

JSV, HM - Drafting the article or revising it critically for important intellectual content

All authors read and approved the final manuscript.

\section{Compliance with Ethical Standards}

Conflict of Interest The authors declare that they have no competing interests.

Informed Consent The authors certify that they have obtained all appropriate patient consent forms. In the form the patient(s) has/have given his/her/their consent for his/her/their images and other clinical information to be reported in the journal, the patients understand that their names and initials will not be published and due efforts will be made to conceal their identity, but anonymity cannot be guaranteed.

\section{References}

1. Altekruse SF, Kosary CL, Krapcho M, Neyman N, Aminou R, Waldron W, editors. SEER cancer statistics review. Bethesda: National Cancer Institute; 1975-2007. http://seer.cancer.gov/csr/ 19752007

2. Burningham Z, Hashibe M, Spector L, Schiffman JD. The epidemiology of sarcoma. Clin Sarcoma Res. 2012;2(1):14.

3. https://www.mohfw.gov.in/. Accessed on 6 June 2020.

4. Wisanuyotin T, Mitchai J, Sirichativapee W, Kosuwon W, Sumnanoont C, Jeeravipoolvarn P. Tissue imprint for intraoperative evaluation of musculoskeletal tumors. J Med Assoc Thail. 2009;92(5):654-9.

5. Bhaker P, Mohan H, Handa U, Kumar S. Role of intraoperative pathology consultation in skeletal tumors and tumor-like lesions. Sarcoma. 2014;2014:902104. https://doi.org/10.1155/2014/ 902104.
6. Bales CE. Laboratory techniques. In: Koss LG, Melamed MR, editors. Diagnostic cytology and its histopathological bases. 5th ed. Philadelphia: Lippincott Williams and Wilkins; 2006. p. 1570-634.

7. Henwood AF. Coronavirus disinfection in histopathology. J Histotechnol. 2020;43(2):102-4. https://doi.org/10.1080/ 01478885.2020.1734718.

8. Coffin CM, Spilker K, Zhou H, Lowichik A, Pysher TJ. Frozen section diagnosis in pediatric surgical pathology: a decade's experience in a children's hospital. Arch Pathol Lab Med. 2005;129(12): 1619-25.

9. Bacci G, Longhi A, Briccoli A, Bertoni F, Versari M, Picci P. The role of surgical margins in treatment of Ewing's sarcoma family tumors: experience of a single institution with 512 patients treated with adjuvant and neoadjuvant chemotherapy. Int $\mathbf{J}$ Radiat Oncol Biol Phys. 2006 Jul 1;65(3):766-72.

10. Kawaguchi N, Matsumoto S, Manabe J. New method of evaluating the surgical margin and safety margin for musculoskeletal sarcoma, analysed on the basis of 457 surgical cases. J Cancer Res Clin Oncol. 1995;121:555-63.

11. Bakhshandeh M, Tutuncuoglu SO, Fischer G, Masood S. Use of imprint cytology for assessment of surgical margins in lumpectomy specimens of breast cancer patients. Diagn Cytopathol. 2007;35(10):656-9.

12. Naveed H, Abid M, Hashmi AA, Edhi MM, Sheikh AK, Mudassir $G$, et al. Diagnostic accuracy of touch imprint cytology for head and neck malignancies: a useful intra-operative tool in resource limited countries. BMC Clin Pathol. 2017;17:25.

13. Chévez-Barrios P, Milman T, Grossniklaus HE, Folberg R, Salomão DR, Gombos DS. Ocular pathology recommendations during COVID-19 from the American Association of Ophthalmic Oncologists and Pathologists (AAOOP). Ocul Oncol Pathol. 2020;6(3):1-2.

14. Compérat E. What does COVID-19 mean for the pathologyurology interaction? Eur Urol. 2020:S0302-2838(20)30216-5. https://doi.org/10.1016/j.eururo.2020.03.041.

Publisher's Note Springer Nature remains neutral with regard to jurisdictional claims in published maps and institutional affiliations. 